\title{
COMPARAÇÃO FLORÍSTICA E ESTRUTURAL DE DUAS FLORESTAS DE VÁRZEA NO ESTUÁRIO AMAZÔNICO, PARÁ, BRASIL ${ }^{1}$
}

\author{
Fábio de Jesus Batista², Mário Augusto Gonçalves Jardim³ ${ }^{3}$ Tonny David Santiago Medeiros ${ }^{3}$ e \\ Isaac Luiz Magalhães Lopes ${ }^{3}$
}

\begin{abstract}
RESUMO - O objetivo deste trabalho foi conhecer e comparar a composição florística e a estrutura de duas áreas de florestas de várzea localizadas na reserva extrativista Chocoaré-Mato Grosso, Santarém Novo-PA. O inventário florístico abrangeu 1,5 ha em parcelas de 10 x 100 m, distribuídas na área 1 (1,0ha) e área 2 (0,5 ha). Foram identificados os indivíduos arbóreos com circunferência a 1,3 m altura do solo (CAP $\geq 30 \mathrm{~cm}$ e demonstrada a riqueza, área basal e o IVI (Índice de Valor de Importância) para cada área. A relação entre as áreas foi realizada por meio da similaridade de espécies, densidade, área basal, análise de agrupamento e espécies indicadoras. Na área 1, ocorreram 613 ind.ha ${ }^{-1}\left(26,67 \mathrm{~m}^{2}\right.$.ha $\left.{ }^{-1}\right)$ distribuídos em 17 famílias, 33 gêneros e 34 espécies com Euterpe oleracea, Enterolobium maximum, Symphonia globulifera, Pterocarpus amazonicus e Virola surinamensis apresentando os maiores IVI's e a área 2 com 744 ind.ha ${ }^{-1}$ (35,34 m².ha $\left.{ }^{-1}\right)$ em 13 famílias, 24 gêneros e 26 espécies com Mauritia flexuosa, Euterpe oleracea, Virola surinamensis, Tapirira guianensis e Inga thibaudiana com os maiores IVI's. As áreas registraram baixas similaridades entre si $(0,18)$ e tanto a densidade quanto a área basal foram superiores na área 2. O agrupamento separou as áreas entre si e das 51 espécies, apenas 15 foram indicadoras. Conclui-se que, as florestas apresentaram baixa riqueza com pouca semelhança entre as populações arbóreas e as espécies indicadoras ocorreram nas áreas 1 e 2.
\end{abstract}

Palavras-chave: Fitossociologia, Reserva extrativista e Santarém Novo.

\section{FLORISTIC AND STRUCTURAL COMPARISON OF TWO FLOODPLAIN FORESTS IN THE AMAZON ESTUARY, PARÁ (PA), BRAZIL}

\begin{abstract}
This study aimed to find and compare the floristic composition and structure of two floodplain forests areas located in the extractive reserve Chocoaré - Mato Grosso, Santarém Novo, PA. The floristic inventory covered 1.5 ha in plots of $10 \times 100 \mathrm{~m}$, distributed in the area 1 (1.0 ha) and area $2(0.5 \mathrm{ha})$. The individual trees with circumference at $1.3 \mathrm{~m}$ height from the soil were identified $(\mathrm{CBH} \geq 30 \mathrm{~cm})$ and demonstrated the richness, basal area, and the Importance Value Index (IVI) in each area. The relationship among the areas was carried out by the species similarity, density, basal area, cluster analysis, and indicator species. In the area 1, there were 613 ind.ha-1 (26.67 m2.ha-1), distributed in 17 families, 33 genera, and 34 species with Euterpe oleracea, Enterolobium maximum, Symphonia globulifera, Pterocarpus amazonicus, and Virola surinamensis with the highest IVIs. In the area 2, with 744 ind.ha-1 (35.34 m2.ha-1) in 13 families, 24 genera, and 26 species with Mauritia flexuosa, Euterpe oleracea, Virola surinamensis, and Tapirira guianensis and Inga thibaudiana with the highest IVIs. The areas recorded low similarities among themselves (0.18), and both the density and basal area were higher in the area 2. The grouping separated the areas among them. Only 15 were indicators from 51 species. It is concluded that the forests had low richness with little resemblance among the tree populations. The indicator species occurred in the areas 1 and 2.
\end{abstract}

Keywords: Phytosociology, Extractive reserve and Santarém Novo.

\footnotetext{
${ }^{1}$ Recebido em 02.12.2008 e aceito para publicação em 16.12.2010.

${ }^{2}$ Secretaria de Estado e Meio Ambiente, SEMA, Brasil. E-mail: <fabiojb@superig.com.br>.

${ }^{3}$ Museu Paraense Emilio Goeldi, MPEG, Brasil.E-mail: <jardim@museu-goeldi.br>, <tjahbio@yahoo.com.br>e

<Isaac_luiz_@hotmail.com>.
} 


\section{INTRODUÇÃO}

Segundo Lugo (1990), as áreas úmidas cobrem no mundo cerca de 250 milhões de hectares, tendo grande importância nos ciclos biogeoquímicos e como habitats para a vida silvestre. Na Região Norte do Brasil, a floresta periodicamente inundada por rios de água branca é conhecida por "floresta de várzea" (GUILLAUMET, 1987) e ocupa cerca de $98.000 \mathrm{~km}^{2} \mathrm{da}$ Amazônia, dos quais $75.880,8 \mathrm{~km}^{2}$ correspondem às florestas de várzea, ou seja, 1,6\% da superfície da Amazônia brasileira (MACEDO et al., 2007).

Em florestas de várzeas localizadas no Estado do Pará, os estudos de comparações florísticas e estruturais até o momento são incipientes e têm demonstrado pouca riqueza e baixa similaridade de espécies (ALMEIDA et al., 2004), porém algumas importantes na subsistência das populações ribeirinhas (JARDIM et al., 2004). Em geral, são poucos estudos realizados em florestas de várzea das unidades de conservação no Pará, a exemplo da Floresta Nacional de Caxiuanã (FERREIRA et al., 1997) e da Área de Proteção Ambiental, Ilha do Combu (JARDIM e VIEIRA, 2001).

Este estudo teve como objetivos conhecer e comparar a composição florística e a estrutura de duas áreas de florestas de várzea localizadas na Resex ChocoaréMato Grosso, Município de Santarém Novo, PA.

\section{MATERIAL E MÉTODOS}

\subsection{Caracterização do Município e das Áreas de Estudo}

O Município de Santarém Novo pertence à mesorregião do Nordeste e à microrregião Bragantina do Estado do Pará, com a sede municipal entre as coordenadas $47^{\circ} 23^{\prime} \mathrm{W}$ e $00^{\circ} 55^{\prime}$ 'S. Segundo a classificação de Köppen, o clima predominante na região é do tipo Am, com reduzida amplitude térmica entre as médias dos meses mais quentes, variando em torno de $26^{\circ} \mathrm{C}$, e a média anual de umidade relativa superior a $80 \%$ (ROCQUE, 1992). Os excedentes hídricos anuais estão entre os meses de fevereiro e junho, e a deficiência hídrica, entre agosto e dezembro, com pluviosidade elevada estando acima de $2.000 \mathrm{~mm}$ (SEPOF, 2005). De acordo com Veloso et al. (1991), baseando-se na altitude e latitude, a vegetação do município pode ser classificada em Floresta Ombrófila Densa, Floresta Ombrófila Aberta e Floresta Ombrófila Densa Aluvial ou esta última como várzea de maré baseada no tipo de rio e intensidade de inundação (PRANCE, 1980).
Os levantamentos de campo foram realizados na Reserva Extrativista (Resex) Chocoaré-Mato Grosso, com 2.785,72 ha em extensão territorial, em duas áreas de florestas de várzea situadas na comunidade Fortaleza, consideradas como áreas 1 e 2 . A distância entre as áreas foi de aproximadamente $1.370 \mathrm{~m}$, com altitude em torno de $14 \mathrm{~m}$. Aárea 1 era composta por extenso fragmento de floresta às margens do rio Chocoaré, situada aproximadamente $350 \mathrm{~m}$ de distância desse curso. É cortada por pequenos igarapés e ladeada por campos, buritizais, florestas secundárias e áreas antropizadas. Sua topografia apresenta suave declividade em direção ao rio. A área 2 é cortada por pequeno fragmento de floresta, ladeada por buritizal, floresta secundária em estágios iniciais de regeneração e áreas antropizadas. Sua topografia é plana e situada na parte inferior de um baixio, motivo pelo qual permanece encharcada durante parte do ano.

\subsection{AMOSTRAGEM E COLETA DE DADOS}

Os inventários foram realizados pelo método de parcelas temporárias (MUELLER-DOMBOIS e ELLENBERG, 1974), em uma área de 1,5 ha dividida e numerada em 15 parcelas de 10 x 100 m (P1 a P15), onde cada parcela foi subdividida em 10 subparcelas de $10 \times 10 \mathrm{~m}$. As parcelas contíguas de P1 a P10 foram instaladas na área 1 (1 ha) e de P11 a P15 na área 2 ( 0,5 ha), sendo todas paralelas ao rio. Aárea 2 apresentou amostragem inferior devido ao tamanho reduzido do fragmento florestal onde as parcelas foram instaladas.

Foram mensurados todos os indivíduos arbóreos com circunferência a $1,3 \mathrm{~m}$ do solo (CAP) $\geq 30 \mathrm{~cm}$. Nas medições dos açaizeiros (Euterpe oleracea Mart.), cada touceira correspondeu a apenas um indivíduo, para que não ocorresse superestimação na abundância da espécie. A identificação das plantas foi realizada em campo por técnicos do Herbário João Murça Pires (MG), do Museu Paraense Emílio Goeldi. Foi coletado material botânico apenas para identificação das espécies que suscitaram dúvidas, e o sistema de classificação utilizado foi Angiosperm Phylogeny Group II (APG II, 2003).

\subsection{ANÁLISE DOS DADOS}

Foram calculados para cada área a densidade e área basal absoluta, riqueza florística, índice de diversidade de Shannon-Wienner (H') (MAGURRAN, 1988), equabilidade (J) (PIELOU, 1977), índice de similaridade de Jaccard entre as parcelas (BROWER et al., 1996), densidade ( $\mathrm{D} \%)$, frequência (Fr\%), dominância (Do\%) e índice de valor de importância 
(IVI) (CURTIS e MCINTOSH, 1951). Para o cálculo da dominância de E. oleracea, utilizaram-se os valores somados de área basal de cada estipe por touceira.

A similaridade florística entre as áreas 1 e 2 foi obtida utilizando o índice de Jaccard (BROWER et al., 1996). A comparação entre as densidades e áreas basais das áreas foi realizada por meio do teste $t$, de Student, para amostras com diferentes tamanhos, a 95\% de probabilidade. Para os testes foi utilizado o programa Bioestat 4.0 (AYRES et al., 2005).

Baseado na distribuição e abundância das espécies, com o intuito de verificar a existência de um mosaico vegetacional entre as florestas, foi realizada a análise de agrupamento, utilizando-se a distância euclidiana como medida de dissimilaridade e o método Ward de agrupamento, por meio do programa PC-ORD 4.0 (MCCUNE e MEFFORD, 1999). Para homogeneização dos dados, fez-se a transformação logarítmica. A partir do dendrograma construído na análise de agrupamento foi aplicada a análise indicadora de espécies, com o índice de valor de indicação da espécie - IndVal, para obter as principais espécies de cada grupo (DUFRÊNE e LEGENDRE, 1997). A significância estatística do IndVal foi avaliada utilizando o teste de randomização de Monte Carlo, com 500 repetições aleatórias e nível de significância de 5\%.

\section{RESULTADOS}

Foi registrado um total de 985 indivíduos, pertencentes a 21 famílias, 45 gêneros e 51 espécies. Das 51 espécies encontradas, 25 foram exclusivas da área 1 e 17 da área 2. As famílias que apresentaram maior riqueza em espécies foram Fabaceae (10), Malvaceae (7) e Clusiaceae (5).

Na área 1, verificou-se a ocorrência de 613 ind.ha-1, com área basal de 26,67 $\mathrm{m}^{2}$.ha-1 , distribuídos em 17 famílias, 33 gêneros e 34 espécies. O valor do índice de diversidade de Shannon foi de 2,58 e a equabilidade foi de 0,73. O índice de Jaccard variou de 0,3 a 0,69, com a menor similaridade entre as parcelas P1 e P10 e a maior similaridade entre as parcelas P7 e P8. As cinco espécies com os maiores IVIs somaram, juntos, 61,9\% do IVI total (Tabela 1). As demais espécies (28), com os menores IVI's, caracterizaram-se por apresentar os menores números de indivíduos e contribuíram com apenas 32,26\% do IVI total. Dez espécies apresentaram somente um indivíduo.
Na área 2 foram registrados 744 ind.ha ${ }^{-1}$, com área basal igual a 35,34 m².ha-1, distribuídos em 13 famílias, 24 gêneros e 26 espécies com índice de diversidade de Shannon de 2,02 e equabilidade de 0,62. O índice de Jaccard variou de 0,33 a 0,53 , com a menor similaridade entre as parcelas P11 e P15 e a maior similaridade entre as parcelas P11 e P12 e P14 e P15. As cinco espécies com os maiores IVIs contribuíram com 82,1\% do IVI total (Tabela 2). Ressalta-se que as demais espécies (21) contribuíram com 17,9\% do IVI total. Nove espécies registraram só um indivíduo.

A similaridade florística entre as áreas 1 e 2 foi igual a 0,18 . Não houve semelhança na composição entre as áreas, pois apenas nove espécies foram comuns, à exceção de C. distachya, C. matourensis e T. guianensis (sendo a última apenas na área 1), todas as demais espécies similares apresentaram posições de destaque na hierarquização do IVI.

As comparações entre densidade ( $p=0,0309)$ e área basal ( $p=0,0176)$ das áreas apresentaram diferenças significativas. A densidade foi maior nas parcelas da área 2 (aproximadamente 744 ind.ha ${ }^{-1}$,

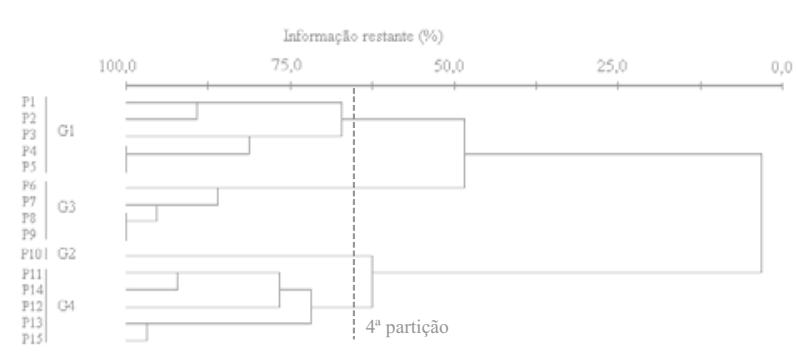

Figura 1- Análise de agrupamento das 15 parcelas amostradas em duas áreas de floresta de várzeas (área 1, P1P10; área 2, P11-P15), Resex Chocoaré-Mato Grosso, Santarém Novo, Pará, por meio da distância Euclidiana, como medida de dissimilaridade e o método Ward de agrupamento. O percentual de encadeamento foi de 6,67. A partição esta indicada pela linha pontilhada (Part.4) e os grupos 1, 2, 3 e 4 por G1, G2, G3 e G4, respectivamente.

Figure 1-Cluster analysis of 15 plots sample in two floodplain forests (area 1, P1-P10; area 2, P11-P15), Chocoaré - Extrative Reserve, Mato Grosso, Santarém Novo, Pará, through Euclidian distance as a dissimilarity measure, and the Ward's grouping method. The percentual chaining was 6,67. The partition is indicating by dotted line (Part. 4) and the groups $1,2,3$, and 4 by $G 1, G 2, G 3$, and G4, respectively.

Revista Árvore, Viçosa-MG, v.35, n.2, p.289-298, 2011 
Tabela 1 - Descritores fitossociológicos das espécies amostradas em 1 ha de floresta de várzea (área 1), Resex ChocoaréMato Grosso, Santarém Novo, Pará. NI=número de indivíduos; NPO=número de parcelas em que ocorreu a espécie; Do=dominância absoluta; Do\%=dominância relativa; D\%=densidade relativa; Fr\%=frequência relativa; e IVI=índice de valor de importância.

Table 1 - Phytosociology descriptors from the sample species in 1 ha of floodplain forest (area 1), Chocoaré - extrative reserve - Mato Grosso, Santarém Novo, Pará. IN = individual number; PNO = plots number of the species occurrence; Do = absolute dominance $;$ Do $\%$ = relative dominance $; \mathrm{D} \%$ = relative density; $F r \%=$ relative frequency; and IVI = importance value index.

\begin{tabular}{|c|c|c|c|c|c|c|c|c|}
\hline Família & Espécie & NI & NPO & Do & Do\% & $\mathrm{D} \%$ & Fr\% & IVI \\
\hline Arecaceae & Euterpe oleracea Mart. & 193 & 71 & 5,03 & 18,86 & 31,48 & 18,78 & 23,04 \\
\hline Fabaceae & Enterolobium maximum Ducke & 63 & 38 & 3,83 & 14,37 & 10,28 & 10,05 & 11,57 \\
\hline Clusiaceae & Symphonia globulifera L. f. & 54 & 32 & 4,55 & 17,06 & 8,81 & 8,47 & 11,44 \\
\hline Fabaceae & Pterocarpus amazonicus Huber & 36 & 26 & 3,99 & 14,97 & 5,87 & 6,88 & 9,24 \\
\hline Myristicaceae & Virola surinamensis (Rol. ex Rottb.) Warb. & 39 & 27 & 1,76 & 6,59 & 6,36 & 7,14 & 6,70 \\
\hline Apocynaceae & Couma utilis (Mart.) Müll. Arg. & 37 & 26 & 1,15 & 4,32 & 6,04 & 6,88 & 5,75 \\
\hline Malvaceae & Matisia paraensis Huber & 21 & 19 & 0,91 & 3,42 & 3,43 & 5,03 & 3,96 \\
\hline Meliaceae & Carapa guianensis Aubl. & 18 & 13 & 0,72 & 2,70 & 2,94 & 3,44 & 3,03 \\
\hline Moraceae & Ficus maxima Mill. & 19 & 15 & 0,41 & 1,54 & 3,10 & 3,97 & 2,87 \\
\hline Arecaceae & Socratea exorrhiza (Mart.) H. Wendl. & 18 & 16 & 0,25 & 0,94 & 2,94 & 4,23 & 2,70 \\
\hline Fabaceae & Pterocarpus officinalis Jacq. & 14 & 13 & 0,43 & 1,62 & 2,28 & 3,44 & 2,45 \\
\hline Arecaceae & Mauritia flexuosa L.f. & 10 & 5 & 1,12 & 4,19 & 1,63 & 1,32 & 2,38 \\
\hline Malvaceae & Sterculia speciosa K. Schum. & 11 & 10 & 0,49 & 1,85 & 1,79 & 2,65 & 2,10 \\
\hline Fabaceae & Inga edulis Mart. & 13 & 13 & 0,17 & 0,63 & 2,12 & 3,44 & 2,06 \\
\hline Annonaceae & Guatteria microsperma R.E.Fries & 14 & 9 & 0,17 & 0,63 & 2,28 & 2,38 & 1,76 \\
\hline Anacardiaceae & Spondias mombin L. & 8 & 6 & 0,25 & 0,93 & 1,31 & 1,59 & 1,28 \\
\hline Cochlospermaceae & Cochlospermum orinocense (Kunth) Steud. & 10 & 5 & 0,10 & 0,39 & 1,63 & 1,32 & 1,11 \\
\hline Sapotaceae & Sarcaulus brasiliensis (A. DC.) Eyma & 6 & 5 & 0,08 & 0,28 & 0,98 & 1,32 & 0,86 \\
\hline Humiriaceae & Sacoglottis guianensis Benth. & 4 & 4 & 0,14 & 0,53 & 0,65 & 1,06 & 0,75 \\
\hline Malvaceae & Bombax paraensis Ducke & 4 & 4 & 0,11 & 0,41 & 0,65 & 1,06 & 0,71 \\
\hline Fabaceae & Machaerium sp. Pers. & 4 & 4 & 0,07 & 0,26 & 0,65 & 1,06 & 0,66 \\
\hline Euphorbiaceae & Hevea brasiliensis (Willd ex A. Juss) Müll. Arg. & 3 & 3 & 0,13 & 0,50 & 0,49 & 0,79 & 0,60 \\
\hline Fabaceae & Campsiandra laurifolia Benth. & 1 & 1 & 0,33 & 1,25 & 0,16 & 0,26 & 0,56 \\
\hline Fabaceae & Erythrina fusca Lour. & 2 & 2 & 0,10 & 0,39 & 0,33 & 0,53 & 0,41 \\
\hline Lecythidaceae & Eschweilera sp. Mart. ex DC. & 1 & 1 & 0,17 & 0,62 & 0,16 & 0,26 & 0,35 \\
\hline Lecythidaceae & Gustavia augusta L. & 2 & 2 & 0,02 & 0,08 & 0,33 & 0,53 & 0,31 \\
\hline Anacardiaceae & Tapirira guianensis Aubl. & 1 & 1 & 0,05 & 0,19 & 0,16 & 0,26 & 0,21 \\
\hline Cecropiaceae & Cecropia distachya Huber & 1 & 1 & 0,04 & 0,15 & 0,16 & 0,26 & 0,19 \\
\hline Sapotaceae & Pouteria guianensisAubl. & 1 & 1 & 0,03 & 0,11 & 0,16 & 0,26 & 0,18 \\
\hline Malvaceae & Quararibea guianensis Aubl. & 1 & 1 & 0,02 & 0,06 & 0,16 & 0,26 & 0,16 \\
\hline Anacardiaceae & Anacardium giganteum W. Hancock ex Engl. & 1 & 1 & 0,01 & 0,04 & 0,16 & 0,26 & 0,16 \\
\hline Euphorbiaceae & Croton matourensis Aubl. & 1 & 1 & 0,01 & 0,03 & 0,16 & 0,26 & 0,15 \\
\hline Simaroubaceae & Simarouba amara Aubl. & 1 & 1 & 0,01 & 0,03 & 0,16 & 0,26 & 0,15 \\
\hline \multirow[t]{2}{*}{ Arecaceae } & Mauritiella armata (Mart.) Burret & 1 & 1 & 0,01 & 0,03 & 0,16 & 0,26 & 0,15 \\
\hline & TOTAL & 613 & - & 26,67 & 99,99 & 100,00 & 100,00 & 100,00 \\
\hline
\end{tabular}

média $=74,4$ ind.parcela ${ }^{-1}$ e s $\left.= \pm 11,74\right)$ do que nas parcelas da área 1 (613 ind.ha ${ }^{-1}$, média $=63,1$ ind.parcela ${ }^{-1}$ e s $= \pm 11,7)$, assim como a área basal, que apresentou maiores valores nas parcelas da área 2 (aproximadamente $35,34 \mathrm{~m}^{2} \cdot$ ha $^{-1}$, média $=3,53 \mathrm{~m}^{2} \cdot$ parcela $^{-1}$ e s $= \pm 0,78$ ) em relação às parcelas da área $1\left(26,67 \mathrm{~m}^{2} \cdot \mathrm{ha}^{-1}\right.$, média $=2,67 \mathrm{~m}^{2}$.parcela ${ }^{-1} \mathrm{e} \mathrm{s}= \pm 0,62$ ). A área 2 diferenciou-se por apresentar, além de E. oleracea, a palmeira $M$. flexuosa com elevada densidade (93 ind. ou 25\% do total) e dominância $\left(9,76 \mathrm{~m}^{2} .0,5 \mathrm{ha}^{-1}\right.$ ou $55,21 \%$ do total).

A análise de agrupamento separou as parcelas nos grupos G1, G2 e G3 correspondentes a área 1 e G4 para área 2 (Figura 1). A análise das espécies indicadoras revelou que das 51 espécies encontradas

Revista Árvore, Viçosa-MG, v.35, n.2, p.289-298, 2011 
Tabela 2 - Descritores fitossociológicos das espécies amostradas em 0,5ha de floresta de várzea (área 2) na Resex ChocoaréMato Grosso, município de Santarém Novo, Pará. NI = número de indivíduos; NPO = número de parcelas em que ocorreu a espécie; Do = dominância absoluta; Do\% = dominância relativa; D\% = densidade relativa; Fr\% = frequência relativa; e IVI = índice de valor de importância.

Table 2 - Phytosociology descriptors from the sample species in 0.5ha of floodplain forest (area 2), Chocoaré - extrative reserve - Mato Grosso, Santarém Novo, Pará. IN = individual number; PNO = plots number of species occurrence; Do = absolute dominance $;$ Do $\%$ = relative dominance $; \%$ = relative density $; \mathrm{Fr} \%=$ relative frequency; and IVI = importance value index.

\begin{tabular}{|c|c|c|c|c|c|c|c|c|}
\hline Família & Espécie & NI & NPO & Do & Do\% & $\mathrm{D} \%$ & Fr\% & IVI \\
\hline Arecaceae & Mauritia flexuosaL.f. & 93 & 31 & 9,76 & 55,21 & 25,00 & 19,25 & 33,16 \\
\hline Arecaceae & Euterpe oleracea Mart. & 137 & 40 & 3,83 & 21,67 & 36,83 & 24,84 & 27,78 \\
\hline Myristicaceae & Virola surinamensis (Rol. ex Rottb.) Warb. & 42 & 24 & 1,75 & 9,92 & 11,29 & 14,91 & 12,04 \\
\hline Anacardiaceae & Tapirira guianensis Aubl. & 19 & 11 & 0,59 & 3,36 & 5,11 & 6,83 & 5,10 \\
\hline Fabaceae & Inga thibaudiana DC. & 15 & 10 & 0,32 & 1,82 & 4,03 & 6,21 & 4,02 \\
\hline Fabaceae & Pterocarpus amazonicus Huber & 13 & 7 & 0,31 & 1,76 & 3,49 & 4,35 & 3,20 \\
\hline Moraceae & Ficus maxima Mill. & 12 & 8 & 0,20 & 1,15 & 3,23 & 4,97 & 3,12 \\
\hline Ebenaceae & Diospyros guianensis (Aubl.) Gürke & 5 & 4 & 0,07 & 0,41 & 1,34 & 2,48 & 1,41 \\
\hline Malvaceae & Apeiba burchellii Sprague & 5 & 2 & 0,14 & 0,78 & 1,34 & 1,24 & 1,12 \\
\hline Clusiaceae & Symphonia globulifera L. f. & 4 & 3 & 0,05 & 0,27 & 1,08 & 1,86 & 1,07 \\
\hline Clusiaceae & Caraipa densifolia Mart. & 3 & 2 & 0,20 & 1,12 & 0,81 & 1,24 & 1,06 \\
\hline Salicaceae & Homalium guianense (Aubl.) Oken & 4 & 2 & 0,07 & 0,38 & 1,08 & 1,24 & 0,90 \\
\hline Euphorbiaceae & Croton matourensis Aubl. & 2 & 2 & 0,08 & 0,44 & 0,54 & 1,24 & 0,74 \\
\hline Euphorbiaceae & Alchorneopsis floribunda (Benth.) Müll. Arg. & 2 & 2 & 0,06 & 0,32 & 0,54 & 1,24 & 0,70 \\
\hline Euphorbiaceae & Richeria Grandis Vahl & 2 & 2 & 0,02 & 0,10 & 0,54 & 1,24 & 0,63 \\
\hline Fabaceae & Tachigalia myrmecophila (Ducke) Ducke & 3 & 1 & 0,03 & 0,16 & 0,81 & 0,62 & 0,53 \\
\hline Clusiaceae & Tovomita brasiliensis (Mart.) Walp. & 2 & 1 & 0,05 & 0,28 & 0,54 & 0,62 & 0,48 \\
\hline Boraginaceae & Cordia tetrandra Aubl. & 1 & 1 & 0,03 & 0,17 & 0,27 & 0,62 & 0,35 \\
\hline Boraginaceae & Cordia scabrifolia A. DC. & 1 & 1 & 0,03 & 0,15 & 0,27 & 0,62 & 0,35 \\
\hline Malvaceae & Sterculia elata Ducke & 1 & 1 & 0,02 & 0,13 & 0,27 & 0,62 & 0,34 \\
\hline Cecropiaceae & Cecropia distachya Huber & 1 & 1 & 0,02 & 0,10 & 0,27 & 0,62 & 0,33 \\
\hline Malvaceae & Bombax longipedicellatum Ducke & 1 & 1 & 0,01 & 0,08 & 0,27 & 0,62 & 0,32 \\
\hline Clusiaceae & Clusia grandiflora Splitg. & 1 & 1 & 0,01 & 0,07 & 0,27 & 0,62 & 0,32 \\
\hline Fabaceae & Inga laurina (Sw.) Willd. & 1 & 1 & 0,01 & 0,05 & 0,27 & 0,62 & 0,31 \\
\hline Bignoniacea & Jacaranda copaia (Aubl.) D. Don & 1 & 1 & 0,01 & 0,05 & 0,27 & 0,62 & 0,31 \\
\hline \multirow[t]{2}{*}{ Clusiaceae } & Vismia guianensis (Aubl.) Pers. & 1 & 1 & 0,01 & 0,04 & 0,27 & 0,62 & 0,31 \\
\hline & TOTAL & 372 & - & 17,67 & 100,00 & 100,00 & 100,00 & 100,00 \\
\hline
\end{tabular}

nas áreas 1 e 2, 36 foram não significativas, 14 significativas e uma altamente significativa em seus valores de IndVal máximo na indicação dos grupos (Tabela 3).

E. oleracea, V. surinamensis, F. maxima, S. globulifera, P. amazonicus e C. matourensis destacaram-se com os maiores valores de IndVal no início do agrupamento (antes da segunda partição do dendrograma), onde as espécies são características das áreas 1 e 2. Com exceção de C. matourensis, todas as outras espécies citadas ocuparam lugares de destaque na hierarquização dos IVIs. As espécies indicadoras da área 1 foram E. maximum, $M$. paraensis, S. exorrhiza, P. officinalis, C. guianensis, I. edulis, S. speciosa, C. orinocense, C. utilis, S. mombin e G. microsperma e da área 2: M. flexuosa, T. guianensis e I. thibaudiana. O grupo G1 foi representado por 13 espécies, o G2 por uma, G3 por sete e G4 por 17. Os grupos G1 e G4 apresentaram os maiores números de espécies indicadoras porque abrangeram cinco parcelas cada. O grupo G2 apresentou característica bem peculiar, a única parcela (P10) da área 1 que registrou a ocorrência de $M$. flexuosa e T. guianensis, espécies consideradas indicadoras da área 2. Esse foi o motivo pelo qual a parcela formou grupo isolado das demais (G1 e G3) e próxima do grupo G4.

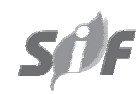

Revista Árvore, Viçosa-MG, v.35, n.2, p.289-298, 2011 
Tabela 3 - Resultado da análise das espécies indicadoras (CAP $\geq 30 \mathrm{~cm}$ ) das florestas de várzea da área 1 e área 2, na Resex Chocoaré-Mato Grosso, município de Santarém Novo, Pará. P = partição; G = grupo (G1, G2, G3 e G4); IndVal= valor de indicação; Rank = classificação; Sig = significância (ns: não significante, *: significante, e **: altamente significante); e P1, ..., e P15 = parcelas estudadas.

Table 3 - Analysis results from indicators species with breast height $>30 \mathrm{~cm}$ ) of two floodplain forests from areas 1 and 2, in Chocoaré - extrative reserve - Mato Grosso, Santarém Novo, Pará. P = partition; G = group (G1, G2, G3, and G4); IndVal = indicating value; Rank = ranking; Sig = significance (ns: no significance, *: significance, and **: high significance); and $\mathrm{P} 1, \ldots$, and $\mathrm{P} 15$ = studies plots.

\begin{tabular}{|c|c|c|c|c|c|c|c|c|c|}
\hline \multirow[t]{3}{*}{$\overline{\mathrm{P}}$} & \multirow[t]{3}{*}{$\bar{G}$} & \multirow[t]{3}{*}{ Espécies } & \multirow[t]{3}{*}{ IndVal } & \multirow[t]{3}{*}{ Sig } & \multirow[t]{3}{*}{ Rank } & \multicolumn{4}{|c|}{ Densidade (ind.área-1) / Frequência } \\
\hline & & & & & & \multicolumn{3}{|c|}{ Área 1 (1 ha) } & \multirow{2}{*}{$\begin{array}{c}\text { Área } 2(0,5 \text { ha }) \\
\text { G4(P11-P15) }\end{array}$} \\
\hline & & & & & & G1(P1-P5) & G2(P6-P9) & G3(P10) & \\
\hline 1 & 1 & Euterpe oleracea & 100 & NS & 500 & $54 / 5$ & $48 / 1$ & $91 / 4$ & $137 / 5$ \\
\hline 1 & 1 & Virola surinamensis & 100 & NS & 500 & $18 / 5$ & $6 / 1$ & $15 / 4$ & $42 / 5$ \\
\hline 1 & 1 & Ficus maxima & 86,67 & NS & 500 & $12 / 4$ & $1 / 1$ & $6 / 3$ & $12 / 5$ \\
\hline 1 & 1 & Symphonia globulifera & 80 & NS & 500 & $33 / 5$ & $5 / 1$ & $16 / 4$ & $4 / 2$ \\
\hline 1 & 1 & Pterocarpus amazonicus & 73,33 & NS & 500 & $7 / 3$ & $8 / 1$ & $21 / 4$ & $13 / 3$ \\
\hline 1 & 1 & Croton matourensis & 13,33 & NS & 500 & $0 / 0$ & $0 / 0$ & $1 / 1$ & $2 / 1$ \\
\hline 2 & 1 & Enterolobium maximum & 97,64 & * & 1 & $47 / 5$ & $1 / 1$ & $15 / 4$ & $0 / 0$ \\
\hline 2 & 1 & Matisia paraensis & 88,89 & * & 2 & $11 / 4$ & $0 / 0$ & $10 / 4$ & $0 / 0$ \\
\hline 2 & 1 & Socratea exorrhiza & 88,89 & * & 4 & $13 / 5$ & $0 / 0$ & $5 / 3$ & $0 / 0$ \\
\hline 2 & 1 & Pterocarpus officinalis & 69,73 & $*$ & 9 & $8 / 4$ & $1 / 1$ & $5 / 3$ & $0 / 0$ \\
\hline 2 & 1 & Sacoglottis guianensis & 33,33 & NS & 98 & $3 / 2$ & $0 / 0$ & $1 / 1$ & $0 / 0$ \\
\hline 2 & 2 & Mauritia flexuosa & 100 & $*$ & 1 & $0 / 0$ & $10 / 1$ & $0 / 0$ & $93 / 5$ \\
\hline 2 & 2 & Tapirira guianensis & 100 & $*$ & 1 & $0 / 0$ & $1 / 1$ & $0 / 0$ & $19 / 5$ \\
\hline 3 & 1 & Carapa guianensis & 95,33 & * & 1 & $17 / 5$ & $1 / 1$ & $0 / 0$ & $0 / 0$ \\
\hline 3 & 1 & Inga edulis & 90,57 & * & 3 & $12 / 5$ & $0 / 0$ & $1 / 1$ & $0 / 0$ \\
\hline 3 & 1 & Sterculia speciosa & 88,89 & $*$ & 1 & $10 / 5$ & $0 / 0$ & $1 / 1$ & $0 / 0$ \\
\hline 3 & 1 & Cochlospermum orinocense & 60 & $*$ & 19 & $10 / 3$ & $0 / 0$ & $0 / 0$ & $0 / 0$ \\
\hline 3 & 1 & Sarcaulus brasiliensis & 48 & NS & 42 & $5 / 3$ & $0 / 0$ & $1 / 1$ & $0 / 0$ \\
\hline 3 & 1 & Erythrina fusca & 40 & $* *$ & 66 & $2 / 2$ & $0 / 0$ & $0 / 0$ & $0 / 0$ \\
\hline 3 & 1 & Gustavia augusta & 40 & NS & 81 & $2 / 2$ & $0 / 0$ & $0 / 0$ & $0 / 0$ \\
\hline 3 & 1 & Hevea brasiliensis & 40 & NS & 82 & $3 / 2$ & $0 / 0$ & $0 / 0$ & $0 / 0$ \\
\hline 3 & 1 & Pouteria guianensis & 20 & NS & 302 & $1 / 1$ & $0 / 0$ & $0 / 0$ & $0 / 0$ \\
\hline 3 & 1 & Campsiandra laurifolia & 20 & NS & 284 & $1 / 1$ & $0 / 0$ & $0 / 0$ & $0 / 0$ \\
\hline 3 & 1 & Anacardium giganteum & 20 & NS & 284 & $1 / 1$ & $0 / 0$ & $0 / 0$ & $0 / 0$ \\
\hline 3 & 1 & Simarouba amara & 20 & NS & 313 & $1 / 1$ & $0 / 0$ & $0 / 0$ & $0 / 0$ \\
\hline 3 & 1 & Quararibea guianensis & 20 & NS & 284 & $1 / 1$ & $0 / 0$ & $0 / 0$ & $0 / 0$ \\
\hline 3 & 3 & Couma utilis & 87,24 & $*$ & 2 & $4 / 2$ & $2 / 1$ & $31 / 4$ & $0 / 0$ \\
\hline 3 & 3 & Spondias mombin & 67,31 & $*$ & 13 & $1 / 1$ & $0 / 0$ & $7 / 3$ & $0 / 0$ \\
\hline 3 & 3 & Guatteria microsperma & 66,18 & $*$ & 16 & $2 / 1$ & $0 / 0$ & $12 / 3$ & $0 / 0$ \\
\hline 3 & 3 & Machaerium sp. & 39,47 & NS & 89 & $1 / 1$ & $0 / 0$ & $3 / 2$ & $0 / 0$ \\
\hline 3 & 3 & Mauritiella armata & 25 & NS & 132 & $0 / 0$ & $0 / 0$ & $1 / 1$ & $0 / 0$ \\
\hline 3 & 3 & Eschweilera sp. & 25 & NS & 129 & $0 / 0$ & $0 / 0$ & $1 / 1$ & $0 / 0$ \\
\hline 3 & 3 & Cecropia distachya & 15 & NS & 268 & $0 / 0$ & $0 / 0$ & $1 / 1$ & $1 / 1$ \\
\hline 4 & 2 & Bombax paraensis & 58,82 & NS & 129 & $1 / 1$ & $1 / 1$ & $2 / 1$ & $0 / 0$ \\
\hline 4 & 4 & Inga thibaudiana & 100 & $*$ & 1 & $0 / 0$ & $0 / 0$ & $0 / 0$ & $15 / 5$ \\
\hline 4 & 4 & Diospyros guianensis & 60 & NS & 73 & $0 / 0$ & $0 / 0$ & $0 / 0$ & $5 / 3$ \\
\hline 4 & 4 & Alchorneopsis floribunda & 40 & NS & 175 & $0 / 0$ & $0 / 0$ & $0 / 0$ & $2 / 2$ \\
\hline 4 & 4 & Homalium guianense & 40 & NS & 175 & $0 / 0$ & $0 / 0$ & $0 / 0$ & $4 / 2$ \\
\hline 4 & 4 & Apeiba burchellii & 40 & NS & 175 & $0 / 0$ & $0 / 0$ & $0 / 0$ & $5 / 2$ \\
\hline 4 & 4 & Richeria grandis & 40 & NS & 188 & $0 / 0$ & $0 / 0$ & $0 / 0$ & $2 / 2$ \\
\hline 4 & 4 & Caraipa densifolia & 40 & NS & 188 & $0 / 0$ & $0 / 0$ & $0 / 0$ & $3 / 2$ \\
\hline 4 & 4 & Clusia grandiflora & 20 & NS & 500 & $0 / 0$ & $0 / 0$ & $0 / 0$ & $1 / 1$ \\
\hline 4 & 4 & Cordia scabrifolia & 20 & NS & 500 & $\underline{0 / 0}$ & $0 / 0$ & $0 / 0$ & $1 / 1$ \\
\hline
\end{tabular}

Revista Árvore, Viçosa-MG, v.35, n.2, p.289-298, 2011 


\begin{tabular}{|c|c|c|c|c|c|c|c|c|c|}
\hline 4 & 4 & Cordia tetrandra & 20 & NS & 500 & $0 / 0$ & $0 / 0$ & $0 / 0$ & $1 / 1$ \\
\hline 4 & 4 & Inga laurina & 20 & NS & 500 & $0 / 0$ & $0 / 0$ & $0 / 0$ & $1 / 1$ \\
\hline 4 & 4 & Vismia guianensis & 20 & NS & 500 & $0 / 0$ & $0 / 0$ & $0 / 0$ & $1 / 1$ \\
\hline 4 & 4 & Bombax longipedicellatum & 20 & NS & 500 & $0 / 0$ & $0 / 0$ & $0 / 0$ & $1 / 1$ \\
\hline 4 & 4 & Tovomita brasiliensis & 20 & NS & 500 & $0 / 0$ & $0 / 0$ & $0 / 0$ & $2 / 1$ \\
\hline 4 & 4 & Jacaranda copaia & 20 & NS & 500 & $0 / 0$ & $0 / 0$ & $0 / 0$ & $1 / 1$ \\
\hline 4 & 4 & Sterculia elata & 20 & NS & 500 & $0 / 0$ & $0 / 0$ & $0 / 0$ & $1 / 1$ \\
\hline 4 & 4 & Tachigalia myrmecophila & 20 & NS & 500 & $0 / 0$ & $0 / 0$ & $0 / 0$ & $3 / 1$ \\
\hline \multicolumn{6}{|c|}{ Densidade Total } & 281 & 85 & 247 & 372 \\
\hline
\end{tabular}

\section{DISCUSSÃO}

E. oleracea apresentou a maior densidade na área 1 (193 ind.ha-1), contribuindo com 31,48\% da densidade total. Essa elevada densidade também foi relatada por Queiroz et al. (2005) e Santos e Jardim (2006). Entretanto, na área 2, além de E. oleracea (137 ind.ha-1), destacou-se $M$. flexuosa por registrar elevados valores de densidade (93 ind.ha-1 ${ }^{-1}$ ), evidenciando que essa área têm maiores condições ambientais favoráveis ao desenvolvimento de palmeiras. Estudos têm relatado que a distribuição, riqueza e abundância das palmeiras são fortemente influenciadas pela umidade do ambiente (PIRES e PRANCE, 1985; JARDIM e VIEIRA, 2001; SALM et al., 2007). Nos estudos de Rabelo et al. (2002), Bentes-Gama et al. (2002a), Almeida et al. (2004) e Queiroz et al. (2005), os valores de áreas basais situaram-se entre 21,3 e 38,65 $\mathrm{m}^{2}$.ha-1 . De fato, as florestas de várzea que sofrem influência de maré são ambientes caracterizados por apresentarem elevada biomassa (PRANCE, 1980).

As duas áreas apresentaram baixa riqueza florística quando comparadas com os estudos de Almeida et al. (2004) ao registrarem 36, 60, 78 e 42 espécies.ha-1 nas Várzeas dos Municípios de Chaves, Afuá, Barcarena e Senador José Porfírio (PA), respectivamente; Santos e Jardim (2006), 70 espécies em 4 ha na floresta de várzea de Santa Bárbara do Pará (PA); Queiroz et al. (2005) 69 espécies distribuídas em 60 gêneros e 29 famílias em 1 ha de floresta de várzea em Bailique (AP), furo do Mazagão (AP) e rio Maniva (PA); e Jardim e Vieira (2001), 45 espécies na várzea baixa e 68 espécies na várzea alta.

Em comparação com outros trabalhos realizados em florestas de várzea estuarina (JARDIM e VIEIRA, 2001; BENTES-GAMA et al., 2002b; ALMEIDA et al., 2004; QUEIROZ et al., 2005; SANTOS e JARDIM, 2006), várias espécies foram relatadas pela primeira vez em várzeas do estuário amazônico, como Guatteria microsperma, Couma utilis, Mauritia armata, Bombax paraensis, Cochlospermum orinocense, Erythrina fusca e Enterolobium maximum na área 1 e Bombax longipedicellatum, Cordia scabrifolia, Caraipa densifolia, Clusia grandiflora, Vismia guianensis, Alchorneopsis floribunda, Homalium guianense e Inga laurina na área 2.

Os valores do índice de diversidade de Shannon corroboram os valores citados para as várzeas da região do estuário, em diferentes localidades do Amapá $\left(H^{\prime}=1,19\right)$ e Pará $\left(H^{\prime}=3,62\right)$ com áreas amostrais de 1 a 14,5 ha (GAMA et al., 2002; BENTES-GAMA et al., 2002a; ALMEIDA et al., 2004; QUEIROZ et al., 2005). Dessa maneira, pode-se sugerir que a diversidade das florestas de várzea da Resex está dentro dos padrões encontrados para essa tipologia florestal. Jardim e Vieira (2001), Gama et al. (2002) eAlmeida et al. (2004) destacaram que as várzeas estuarinas são caracterizadas pela presença de oligoespécies, isto é, espécies que apresentam elevado número de indivíduos por unidade de área. Essa presença também foi observada nas áreas 1 e 2 , e a característica ecológica para explicar esse comportamento, provavelmente, envolve questões referentes à adaptação das espécies.

A baixa similaridade florística entre as florestas de várzea das áreas 1 e 2, segundo Almeida et al. (2004), é, no entanto, decorrente da imensa variação nos rios, ilhas e lagos, como os efeitos de zonação, altura de inundação, salinidade e velocidade da água.

Entre as espécies que foram comuns às áreas, E. oleracea ocorreu bem distribuída e abundantemente. Esse resultado também foi obtido por Jardim et al. (2004), Jardim e Vieira (2001) e Almeida et al. (2004), ao estudarem diferentes florestas de várzea do estuário amazônico.

Revista Árvore, Viçosa-MG, v.35, n.2, p.289-298, 2011 
De acordo com Gama et al. (2005), as espécies arbóreas requerem um habitat específico e a maior parte delas um muito específico, determinado pelas condições climáticas e edáficas. A densidade dessa espécie talvez seja regulada mais por fatores independentes (clima, solo etc.) do que dependentes (relações biológicas).

A formação dos grupos na análise de agrupamento reforçou ainda mais a suposição de que essas áreas são compostas por comunidades distintas. Mas a divisão da área 1 em três grupos demonstrou que a diferença na riqueza e abundância das populações formou uma área heterogênea, com gradiente alterando na mesma direção das parcelas. Certamente, essa constatação ocorreu devido às pequenas variações ambientais entre as parcelas, como a diferença entre os níveis e o tempo de inundação denominado por Bertani et al. (2001) heterogeneidade ambiental.

Essa heterogeneidade tem sido apontada em vários estudos relacionados ao ambiente de floresta de várzea e é atribuída a uma série de fatores, como a variação da várzea de maré, gerando várias subdivisões vegetacionais (PRANCE, 1980); a capacidade dos vegetais de se adaptarem às mudanças periódicas dos níveis de inundação (JUNK, 1980); a distância entre as áreas (ALMEIDA et al., 2004); a presença de espécies com considerável número de indivíduos por unidade de área (JARDIMe VIEIRA, 2001;ALMEIDAetal., 2004; SANTOS e JARDIM, 2006) e as inundações periódicas, apesar de restritivas em termos de diversidade, têm atuação diferenciada no espaço (BERTANI et al., 2001).

Poucas espécies de várzea apresentam ampla distribuição de ocorrência na região do estuário (ALMEIDA et al., 2004). Das seis espécies mais abundantes e frequentes da várzea das áreas 1 e 2 estão E. oleracea, P. amazonicus, S. globulifera e $V$. surinamensis, que também apresentam as maiores distribuições nos Estados do Amapá e Pará (JARDIM e VIEIRA, 2001; BENTES-GAMA et al., 2002a; ALMEIDA et al., 2004; QUEIROZ et al., 2005; SANTOS e JARDIM, 2006).

O fato de as áreas 1 e 2 serem compostas por fragmentos florestais desperta atenção para realização de estudos dos fragmentos quanto ao tamanho, forma, presença de cursos de água e efeito de borda, pois, segundo Borges et al. (2004), pequenos fragmentos apresentam frágeis padrões de sustentabilidade ao longo do tempo. Os referidos autores complementaram ainda que, para protegê-los da ação antrópica e do efeito de borda, é preciso promover educação ambiental, restringir a presença de animais domésticos na área, implementar corredores com vegetação e aumentar o tamanho dos fragmentos.

\section{CONCLUSÃO}

As áreas de florestas de várzea apresentam baixa riqueza florística com a presença de espécies indicadoras que podem ser característica inerente à tipologia vegetal ou resultado do impacto da ação antrópica nas áreas. A ocorrência das espécies indicadoras em áreas fragmentadas representa informação valiosa para o manejo em programas de recuperação desses ambientes.

\section{AGRADECIMENTOS}

Ao Programa de Pesquisa em Biodiversidade (PPBIO) do Ministério do Meio Ambiente - Temático Bioprospecção, pelo apoio financeiro; a Isaac Loureiro, representante da Prefeitura Municipal de Santarém Novo, pela contribuição; e a todos os moradores da localidade Fortaleza, pelo apoio.

\section{REFERÊNCIAS}

ALMEIDA, S. S.; AMARAL, D. D.; SILVA, A. S. Análise florística e estrutura de florestas de várzea no estuário amazônico. Acta Amazônica, v.34, n.4, p.513-524, 2004.

APG II. An update of the Angiosperm Phylogeny Group classification for the orders and families of flowering plants: APG II. Botanical Journal of the Linnean Society, v.141, p.399-436, 2003.

AYRES, M.; JÚNIOR, M. A.; AYRES, D. L.; SANTOS, A. S. Bioestat 4.0 - Aplicações Estatísticas nas Áreas das Ciências Biológicas e Médicas. Belém: Sociedade Civil Mamirauá/MCT/Imprensa Oficial do Estado do Pará, 2005. 324p.

BENTES-GAMA, M. M.; SCOLFORO, J. R. S.; GAMA, J. R. V. Potencial produtivo de madeira e palmito de uma floresta secundária de várzea baixa no estuário amazônico. Revista Árvore, v.26, n.3, p.311-319, 2002a.

BENTES-GAMA, M. M. et al. Estrutura e valoração de uma floresta de várzea alta na Amazônia. Cerne, v.8, n.1, p.88-102, 2002b. 
BERTANI, D. F. et al. Análise temporal da heterogeneidade florística e estrutural em uma floresta ribeirinha. Revista Brasileira de Botânica, v.24, n.1 p.11-23, 2001.

BORGES, L. F. R. et al. Inventário de fragmentos florestais nativos e proposta para seu manejo e o da paisagem. Cerne, v.10, n.1, p.22-38, 2004.

BROWER, J. E.; ZAR, J. H.; von ENDE, C. N.. Field and laboratory methods for general ecology. 3.ed. Dubuque: Brown Publishers, 1996. 237p.

CURTIS, J.T.; MCINTOSH, R.P. The interrelations of certain analytic and synthetic

phytosociological charaters. Ecology, v.31, n.3, p.476-496, 1951.

DUFRÊNE, M.; LEGENDRE, P. Species assemblages and indicator species: the need for flexible asymmetrical approach. Ecological Monographs, v.67, n.3, p.345-366, 1997.

FERREIRA, L. V.; ALMEIDA, S. S.; ROSÁRIO, C. S. Àreas de inundação. In: LISBOA, P.L.B. (org.) Caxiuanã. Belém: Museu Paraense Emilio Goeldi, 1997. p.195-211.

GAMA, J. R. V. et al. Comparação entre florestas de várzea e de terra firme do Estado do Pará.

Revista Árvore, v.29, n.4, p.607-616, 2005.

GAMA, J. R. V. BOTELHO, S. A.; BENTESGAMA, M. M. Composição florística e estrutura da regeneração natural de floresta secundária de várzea baixa no estuário amazônico. Revista Árvore, v.26, n.5, p.559-566, 2002.

GUILLAUMET, J. L. Some structural and floristic aspect of the forest. Experientia, v.43, p.241-251, 1987.

INSTITUTO BRASILEIRO DE GEOGRAFIA E ESTATÍSTICA - IBGE. Censo demográfico 2000. Rio de Janeiro: 2000. 520p.

JARDIM, M. A. G. et al. Análise florística e estrutural para avaliação da fragmentação nas florestas de várzea do estuário amazônico. In: JARDIM, M.A.G.; MOURÃO, L.; GROISSMAN, M. (Orgs.) Açaí possibilidades e limites para o desenvolvimento sustentável no estuário amazônico. Belém: Museu Paraense Emilio Goeldi, 2004. p.101-121.
JARDIM, M. A. G.; VIEIRA, I. C. G. Composição florística e estrutura de uma floresta de várzea do estuário amazônico, ilha do Combu, Estado do Pará, Brasil. Boletim do Museu Paraense Emilio Goeldi - Série Botânica, v.17, n.2, p.333-354, 2001.

JUNK, W. J. Áreas inundáveis - Um desafio para Limnologia. Acta Amazonica, v.10, n.4, p.775-795, 1980.

LUGO, A. E. Fringe wetlands. In: LUGO, A. E.; BRINSON M.; BROWN S. (Eds.) Forested wetlands. Ecosystems of the world 15. Amsterdam: Elsevier, 1990. p.143-169.

MACEDO, D. S. et al. Produção madeireira, comercialização e o potencial para a certificação florestal nas várzeas: perspectivas para o novo milênio. In: SALOMÃO, R. P.; TEREZO, E. F. M.; JARDIM, M. A. G. Manejo florestal nas várzeas: oportunidades e desafios. Belém: Museu Paraense Emilio Goeldi, 2007. p.139-175.

MAGURRAN, A. E. Ecological diversity and its measurement. Princenton Univ. Press. 1988. p.179.

MCCUNE, B.; MEFFORD, M. J. PC-Ord for Windows: multivariate analysis of ecological: data. Version 4.10. Oregon: MjM Software Design, Gleneden Beach, 1999. 47p.

MUELLER-DOMBOIS, D.; ELLENBERG, H. Aims and methods of vegetation ecology. New York: John Wiley \& Sons, 1974. p.546.

PIELOU, E. C. Mathematical ecology. New York: Wiley, 1977. p.165.

PIRES, J. M.; PRANCE, G. T. The vegetation types of the Brasilian Amazon. In: PRANCE, G. T. (Ed.). Amazonian. New York: Pergamon Press, 1985. p.109-145.

PRANCE, G. T. A terminologia dos tipos de florestas amazônicas sujeitas à inundação. Acta Amazônica, v.10, n.3, p.495-504, 1980.

Revista Árvore, Viçosa-MG, v.35, n.2, p.289-298, 2011 
QUEIROZ, J. A. L. et al. Composição florística e estrutura de floresta em várzea alta estuarina Amazônica. Floresta, v.35, n.1, p.41-55, 2005.

RABELO, F. G. et al. Diversidade, composição florística e distribuição diamétrica do povoamento com DAPe” $5 \mathrm{~cm}$ em região do estuário do Amapá. Revista de Ciências Agrárias, v.37, n.37, p.91-112, 2002.

ROCQUE, C. História dos Municípios do Estado do Pará. Belém: IBGE, 1992. v.1. 280p.

SALM, R. et al. Cross-Scale determinants of palm species distribution. Acta Amazonica, v.37, n.1, p.17-26, 2007.
SANTOS, G. C.; JARDIM, M. A. G. Florística e estrutura do estrato arbóreo de uma floresta de várzea no município de Santa Bárbara do Pará, Estado do Pará, Brasil. Acta Amazonica, v.36, n.4, p.437-446, 2006.

PARÁ. Secretaria Executiva de Estado de Planejamento, Orçamento e Finanças. Estatística Municipal: Santarém Novo. Belém: SEPOF. 2005. p.37.

VELOSO, H. P.; RANGEL FILHO, A. L. R.; LIMA, J.C.A. Classificação da vegetação brasileira adaptada a um sistema universal. Rio de Janeiro: IBGE, 1991. p.124. 